\title{
Tratamiento primario de aguas almacenadas en estanques rústicos mediante la aplicación de coagulantes químicos y biológicos
}

\section{Primary treatment of water stored in rustic ponds through the application of chemical and biological coagulants}

Gemma Arintzy Moreno-
Cabrera ${ }^{1}$,
Gustavo Alvarez-Arteaga ${ }^{1 *}$ [,
María Estela Orozco-
Hernández ${ }^{10}$,
María Antonieta Reyes-
Zuazo ${ }^{1}$
'Facultad de Planeación Ur-
bana y Regional. Universidad
Autónoma del Estado de
México. Instituto Literario No.
100. Col. Centro. CP. 50000.
Toluca, Estado de México,
México.
*Autor de correspondencia:
galvareza@uaemex.mx

Nota cientifica

Recibida: 09 de octubre de 2020

Aceptada: 29 de julio 2021

Como citar: Moreno-Cabrera GA, Alvarez-Arteaga

Orozco-Hernández ME, ReyesZuazo MA (2021) Tratamiento primario de aguas almacenadas en estanques rústicos mediante la aplicación de coagulantes químicos y biológicos. Ecosistemas y Recursos Agropecuarios 8(2): e2734. DOI: 10.19136/era.a8n2.2734
RESUMEN. El estudio evaluó el efecto coagulante del extracto de la semilla de Moringa oleífera solo o en combinación con sulfato de aluminio para el tratamiento de aguas almacenadas en un estanque rústico. Se probaron cuatro tratamientos a partir del uso individual y combinado del extracto con sulfato de aluminio a los 60, 120 y 180 minutos de sedimentación. Los tratamientos combinados tuvieron remociones superiores al $95 \%$ de turbiedad a los primeros 60 minutos en tanto que la aplicación de Moringa oleífera al 100\% tuvo una remoción máxima del 90\% hasta los 180 minutos. En los tratamientos con sulfato de aluminio hubo disminución del $\mathrm{pH}$ en el agua. La combinación del extracto de semillas de Moringa oleífera y sulfato de aluminio en relación $\mathrm{p} / \mathrm{p}$ de $70 / 30$ presentó la mayor eficiencia en remoción y tiempo de sedimentación, con la ventaja de reducir costos y disminuir los sedimentos con aluminio.

Palabras clave: Coagulante biológico, Moringa oleifera, sulfato de aluminio, turbiedad, tratamiento de aguas.

ABSTRACT. The study evaluated the coagulant effect of Moringa oleifera seed extract alone or in combination with aluminium sulphate for the treatment of stored water in a rustic pond. Four treatments were tested from the individual and combined use of the extract with aluminium sulphate at 60,120 and 180 minutes of sedimentation. The combined treatments had removals greater than $95 \%$ of turbidity in the first 60 minutes, while the application of Moringa oleifera at $100 \%$ had a maximum removal of $90 \%$ up to 180 minutes. In the aluminium sulphate treatments there was a decrease in the $\mathrm{pH}$ in the water. The combination of Moringa oleifera seed extract and aluminium sulphate in a 70/30 $\mathrm{w} / \mathrm{w}$ ratio presented the highest removal efficiency and sedimentation time, with the advantage of reducing costs and sediments with aluminium.

Key words: Biological coagulant, Moringa oleifera, aluminium sulphate, turbidity, water treatment. 


\section{INTRODUCCIÓN}

Cifras de la Organización Mundial de la Salud indican que cerca de 2100 millones de personas carecen de agua potable en sus hogares y más del doble no dispone de saneamiento seguro debido a la carencia de infraestructura básica (OMS 2017). En diversas regiones del mundo, la captación de agua pluvial mediante almacenes rústicos artificiales es la principal alternativa para proveer de este líquido a pequeñas comunidades rurales, pero su calidad con frecuencia no cumple con los requisitos sanitarios mínimos para el consumo humano, debido a la elevada concentración de partículas suspendidas y agentes patógenos, representando en consecuencia un problema de salud pública local (Cardozo et al. 2013).

Para remover las partículas suspendidas en el agua se emplean métodos de coagulación que consisten en la adición de compuestos químicos y/o biológicos, para desestabilizar las partículas presentes y aglutinarlas en flóculos con un peso específico mayor al del agua, por lo que al sedimentarse pueden removerse (Mani et al. 2019). Los coagulantes comúnmente empleados en países en vías de desarrollo son la cal, el cloruro férrico, el ácido sulfúrico, poli electrólitos y el sulfato de aluminio (Saritha et al. 2017), siendo este último el más empleado por su eficiencia y relativa economía (Sandoval y Laines 2013); no obstante, algunos estudios relacionan la acumulación de residuos de aluminio en los lodos de sedimentación con la prevalencia de trastornos neuronales como el Alzheimer y Parkinson que disminuyen la capacidad motora y mental (Rondeau et al. 2000, Wang et al. 2016, Bondy 2016). Alternativamente, se han propuesto tratamientos para el saneamiento de aguas mediante el empleo de compuestos vegetales biodegradables, ya sea de manera separada o bien mezclados con sales minerales, como los polisacáridos provenientes de Cactus lefaria, Opuntimma ficus-indica, Plantago psyllium, Malva sylvestris, Hibiscus esculentus y Moringa oleífera Lam (Yin 2010, Sotheeswaran et al. 2011, Feria et al. 2014, Mera et al. 2016). En ambos casos se han obtenido resultados satisfactorios en términos de coagulación, disponibilidad y economía (Dalen et al. 2010, Shan et al. 2017, Cardozo et al. 2018).

Uno de los coagulantes naturales más empleados proviene del extracto de la semilla de M. oleifera, que contiene cantidades considerables de aminoácidos polares con carga neta positiva y negativa (Villaseñor et al. 2018, Ferreira 2020). Estas moléculas pueden interactuar con las partículas coloidales responsables de la turbiedad y el color durante el proceso de clarificación de las aguas, contribuyendo de esta forma a la eliminación de las mismas (Campos et al. 2003), su empleo ha sido documentado en múltiples trabajos (Mendoza et al. 2000, Santana et al. 2010, Delelegn et al. 2018). Una ventaja adicional es la plasticidad genética de la planta para tolerar condiciones de sequía y altas temperaturas, pudiendo cultivarse bajo diferentes condiciones de clima y suelos, lo cual favorece la disponibilidad local de la semilla (Olson y Fahey 2011). El extracto de semillas de $M$. oleifera ha sido aplicado bajo diferentes formas y concentraciones, encontrando resultados variables en su efecto coagulante cuando se aplica solo (Mendoza et al. 2000, Rondón et al. 2017), o bien en combinación con otros coagulantes como el sulfato de aluminio (Sandoval y Laines 2013). En ambas condiciones se han obtenido eficiencias superiores al $90 \%$ en la reducción de la turbiedad. Por su parte, Ndabigensere y Narasiah (1996), determinaron que la producción de lodo residual es menor a la obtenida con sulfato de aluminio, pudiendo ser empleados como abono orgánico, sin contaminar los suelos. Bajo este enfoque, el objetivo de este estudio fue comparar la eficiencia de remoción de la turbiedad en el agua a partir del uso de semillas de M. oleifera y sulfato de aluminio en forma separada y en combinación a diferentes concentraciones.

\section{MATERIALES Y MÉTODOS}

\section{Toma de muestras}

Las muestras de agua procedieron de un estanque artificial con una profundidad promedio de $2.5 \mathrm{~m}$, localizado en una finca rural del municipio de Zirándaro, Guerrero. El agua que ingresa al estanque 
proviene de los escurrimientos superficiales intermitentes, que son recolectados durante los meses de junio a noviembre. Al momento de la toma de muestra de agua (obtenida a $1 \mathrm{~m}$ de profundidad), en el mes de febrero de 2018, se determinaron mediante un equipo multiparámetro marca Hanna Instruments H196130 los siguientes parámetros: pH (9.5), conductividad eléctrica (22 $\left.\mu \mathrm{cm}^{-1}\right)$, turbiedad (61 NTU), sólidos totales disueltos $(0.30 \mathrm{ppm})$, temperatura (28 $\left.{ }^{\circ} \mathrm{C}\right)$ y oxígeno disuelto $\left(20 \mathrm{mg} \mathrm{L}^{-1}\right)$. Posteriormente se trasladó a laboratorio para los estudios de floculación.

\section{Recolección y preparación del extracto de semillas}

Para la preparación de los tratamientos se cosecharon semillas maduras de $M$. oleífera obtenidas de una plantación local, adyacente al estanque; éstas se descascararon y secaron a temperatura ambiente $(25 \circ \mathrm{oC})$ por $24 \mathrm{~h}$, para luego triturarlas en mortero de porcelana y pasarlas por un tamiz de $2 \mathrm{~mm}$ de apertura. A partir del tamizado se tomaron $20 \mathrm{~g}$ para proceder a la extracción de lípidos, realizada en el Laboratorio de servicios externos de la Facultad de Química UAEMéx, considerando la normatividad vigente (SSA 2000).

Para la preparación de las soluciones coagulantes, se empleó la metodología propuesta por Sandoval y Laines (2013). Se requirieron $4 \mathrm{~g}$ de harina de semilla de $M$. oleífera libre de aceites y 4 $\mathrm{g}$ de sulfato de aluminio para la preparación de las diferentes mezclas experimentales de acuerdo a los siguientes tratamientos: a) Control (C) sin floculante; b) SA $100 \%$ ( $2 \mathrm{~g}$ de sulfato de aluminio), c) MO 100 (2 g M. oleífera); d) SAMO 70/30 (1.5 g de sulfato de aluminio / $0.5 \mathrm{~g}$ de M. oleífera); e) SAMO 30/70 (0.5 g de sulfato de aluminio / $1.5 \mathrm{~g}$ de $M$. oleífera). Las mezclas se diluyeron en $50 \mathrm{~mL}$ de agua destilada, fueron agitadas por 15 min a $100 \mathrm{rpm}$ y se depositaron en frascos ámbar de $250 \mathrm{~mL}$ debidamente identificados.

\section{Test de jarras}

Mediante la prueba del test de jarras se procedió a determinar el tratamiento coagulante óptimo, considerando un diseño experimental al azar con una solución testigo y cuatro tratamientos experimentales con cinco repeticiones por tratamiento $(n=25)$. A cada vaso se le agregaron $500 \mathrm{~mL}$ de agua y 2.5 $\mathrm{mL}$ de la solución tratamiento. Para homogeneizar las muestras, se utilizó una placa de agitación marca Corning a $150 \mathrm{rpm}$ durante 3 minutos. Se midieron los valores de turbiedad, $\mathrm{pH}$, temperatura, conductividad eléctrica a los 60, 120 y 180 minutos, y alcalinidad a los 180 minutos. El análisis estadístico se realizó mediante el software SPSS 17, aplicando las pruebas de ANOVA de una vía para probar la igualdad de medias entre tratamientos y la comparación de medias de Tukey $(p>0.01)$ para determinar diferencias significativas entre tratamientos.

\section{RESULTADOS Y DISCUSIÓN}

\section{Remoción de la turbiedad}

Se identificaron diferencias estadísticas significativas ( $p>0.01)$ entre los tratamientos y tiempos de sedimentación (Tabla 1). A los 60 minutos, los tratamientos SAMO 70/30 y SAMO 30/70 tuvieron la mayor eficiencia de remoción de la turbiedad con valores del 98.8 y $95 \%$, respectivamente, mientras que la eficiencia fue menor cuando ambos coagulantes se emplearon de forma separada (<90\%), presentando el menor desempeño SA 100, lo cual puede explicarse a partir estudios que demuestran su mayor eficiencia con un pH entre 5.5 y 8.0 (Ferreira 2020). Considerando que el $\mathrm{pH}$ de la muestra inicial de agua es superior a 9, es probable que ello influyera en la disminución de la eficiencia de coagulación de este agente químico.

A los 120 y 180 minutos de sedimentación, los tratamientos SA 100, SAMO 70/30 y SAMO 30/70 tuvieron eficiencias superiores al $98 \%$, este dato es consistente con lo reportado por Dalen et al. (2010) y Cardozo et al. (2013), quienes indican que la mezcla de $M$. oleifera con soluciones salinas mejora el efecto de coagulación debido al incremento de la solubilidad de las proteínas por acción de las sales. En el presente estudio, el mejor desempeño de $M$. oleifera como coagulante único se obtuvo hasta los 180 minutos, por lo que se corrobora el supuesto 
anterior. Cabe destacar que a lo largo del experimento, la eficiencia de coagulación del tratamiento MO 100 fue significativamente inferior a los demás tratamientos experimentales. De acuerdo a los niveles establecidos por la normatividad mexicana (SSA 2000), los tratamientos SA100, SAMO 70/30 y SAMO 30/70 cumplen con el requerimiento límite permisible de 5 NTU de turbiedad, no así el tratamiento MO, que finaliza a los 180 minutos con 10.60 UTN.

Tabla 1. Valores de turbiedad y porcentaje de remoción en las muestras de agua a diferentes tiempos de exposición al floculante.

\begin{tabular}{|c|c|c|c|c|}
\hline \multirow[b]{3}{*}{ Tratamiento } & \multicolumn{4}{|c|}{ Tiempo (minutos) } \\
\hline & 0 & 60 & 120 & 180 \\
\hline & \multicolumn{4}{|c|}{ Turbiedad (NTU) / Remoción (\%) } \\
\hline \multirow[t]{2}{*}{$\mathrm{C}$} & 150.00 & $39.9^{a}$ & 17.8 & 18.1 \\
\hline & & 73.4 & 88.1 & 88.0 \\
\hline \multirow[t]{2}{*}{ SA-100 } & 253.40 & $88.4^{a}$ & $3.5^{c}$ & $2.4^{c d}$ \\
\hline & & 62.1 & $98.6^{a}$ & $99.1^{a}$ \\
\hline \multirow[t]{2}{*}{ MO-100 } & 101.66 & $14.8^{b}$ & $13.3^{b}$ & $10.6^{b}$ \\
\hline & & $85.4^{a}$ & 87.0 & 89.6 \\
\hline \multirow[t]{2}{*}{ SAMO $70 / 30$} & 133.00 & $1.6^{b}$ & $1.5^{c}$ & $1.1^{c d}$ \\
\hline & & 98.8 & 98.9 & 99.2 \\
\hline \multirow[t]{2}{*}{ SAMO $30 / 70$} & 127.60 & $6.4^{b}$ & $5.2^{c}$ & $4.52^{c}$ \\
\hline & & 95.0 & 95.9 & 96.50 \\
\hline
\end{tabular}

C: Tratamiento control, SA: sulfato de aluminio, MO: extracto de semilla de $M$. oleifera. Medidas con letras iguales dentro de cada columna no difieren estadísticamente (Tukey, $p<0.01$ )

\section{Efectos sobre otros parámetros fisicoquímicos en el agua}

Resultó evidente que en aquellos tratamientos en los que se empleó sulfato de aluminio a cualquier concentración, se observaron efectos significativos sobre la reducción del $\mathrm{pH}$ del agua con respecto al testigo (Figura 1), denotando que a mayor concentración de sulfato de aluminio, se incrementó el efecto acidificante en el agua. El tratamiento MO 100 no registró diferencias significativas y conservó su pH próximo al inicial. Para MO 100 no se reporta pérdida de eficiencia relacionada con $\mathrm{pH}$, pero la eficiencia de remoción de la turbiedad (85\%), fue menor a la reportada en estudios consultados (Cardozo et al. 2013, Sandoval y Laines 2013).

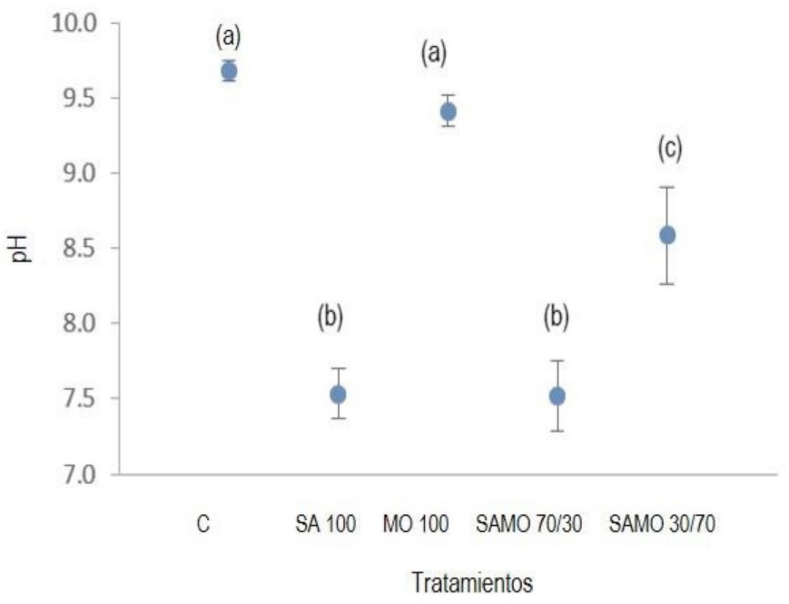

Figura 1: Valores promedio ( $\pm \mathrm{DS}$ ) del pH para los diferentes tratamientos. Letras diferentes indican diferencias en los tratamientos (Tukey, $\mathrm{p}<$ $0.01)$.

La conductividad eléctrica (Figura 2), se situó entre 0.2 y $0.42 \mathrm{mS} \mathrm{m}^{-1}$. El análisis estadístico de los datos no denotó diferencias significativas entre tratamientos, excepto para SAMO 70/30. Las pruebas de alcalinidad obtenidas únicamente al termino de los 180 minutos (Figura 3), indican una disminución significativa para el tratamiento SAMO $70 / 30\left(72+3.16 \mathrm{mg} \mathrm{L}^{-1}\right)$, sobre los tratamientos restantes. Con respecto a estos parámetros, los valores observados se sitúan dentro de los rangos permisibles por la normatividad vigente, por lo que ninguno de los tratamientos empleados altera su condición.

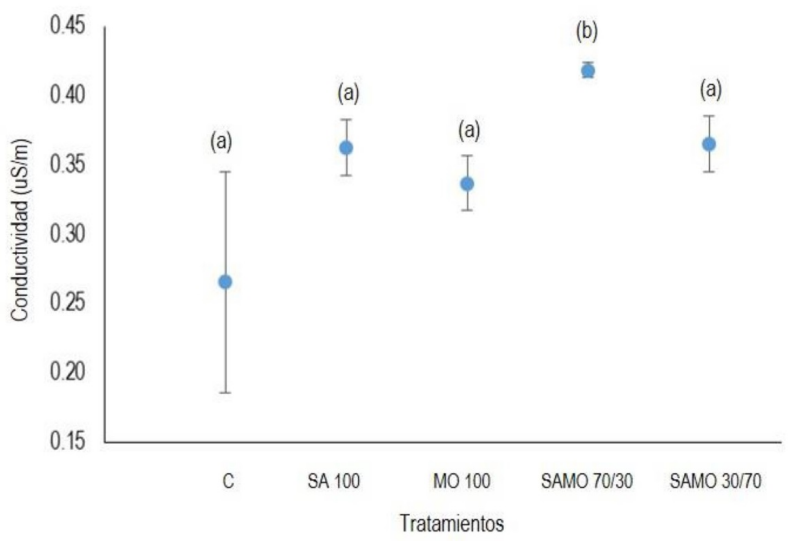

Figura 2: Valores promedio ( \pm DS) de la conductividad eléctrica para los diferentes tratamientos y tiempos de sedimentación. Letras diferentes indican diferencias en los tratamientos (Tukey, $p<0.01$ ). 


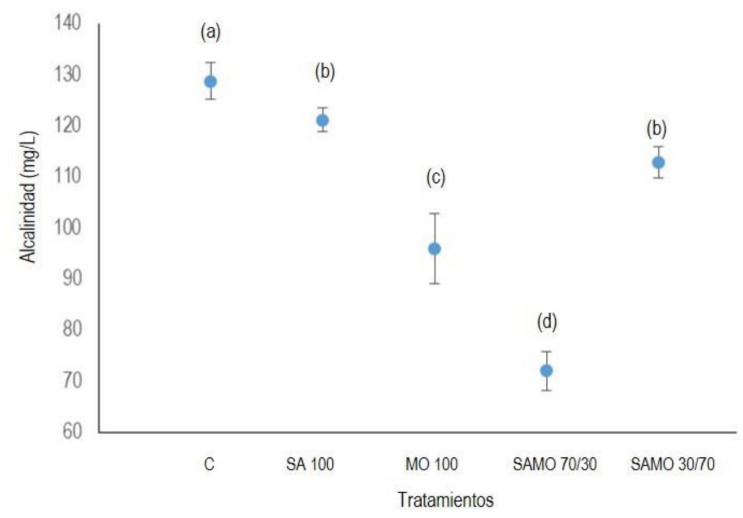

Figura 3: Valores promedio ( $\pm \mathrm{DS}$ ) de alcalinidad en los tratamientos experimental a los 180 minutos. Letras diferentes indican diferencias en los tratamientos (Tukey, $\mathrm{p}<0.01$ ).

Considerando los resultados del estudio, la combinación del extracto de semillas de $M$. oleifera y sulfato de aluminio proporcionan la mayor eficiencia en términos de remoción de partículas suspendidas (> 95\%) y tiempo de floculación (60 minutos). La aplicación individual del extracto de semillas de $M$. oleifera tuvo un efecto coagulante significativamente inferior $(<90 \%)$ y mayor tiempo de sedimentación (180 minutos), mientras que el máximo efecto para el sulfato de aluminio se consiguió a los 120 minutos de sedimentación. Bajo esta perspectiva, la mezcla de ambos agentes coagulantes tiene dos importantes connotaciones: reducir el tiempo del proceso de coagulación-sedimentación y mejorar el efecto individual de ambos agentes. De manera alternativa, es factible reducir el consumo de sulfato de aluminio hasta en un $75 \%$ con respecto a su aplicación habitual, disminuyendo tanto los costos como el daño potencial a la salud humana por su acumulación en los sedimentos.

\section{AGRADECIMIENTOS}

Al apoyo recibido por parte del personal adscrito a la Unidad de Laboratorio de Ciencias ambientales, Facultad de Planeación Urbana y Regional de la Universidad Autónoma del Estado de México.

\section{LITERATURA CITADA}

Bondy SC (2016) Low levels of aluminum can lead to behavioural and morphological changes associated with Alzheimer's disease and age-related neurodegeneration. Neurotoxicology 52: 222-229.

Campos SX, Di Bernardo L, Vieira EM (2010) Influencia das características das substáncias húmicas na eficiencia da coagulacáo com sulfato de aluminio. Engenharia Sanitária e Ambiental 10: 194-199.

Cardoso K, Konradt C, Carvalho M, Pereira F, Bergamasco R (2013) Coagulation diagram using the Moringa oleifera Lam and the aluminium sulphate, aiming the removal of colour and turbidity of water. Acta Scientiarum. Technology 5: 485-469.

Cardoso K, Ferri P, Fernández M, Nishi L, Carvalho M, Bergamasco R (2018) Moringa oleifera Lam and its potential association with aluminium sulphate in the process of coagulation/flocculation and sedimentation of surface water. International Journal of Chemical Engineering 2018: 1-6.

Dalen MB, Pam JS, Izang A, Ekele R (2010) Synergy between Moringa oleifera seed powder and alum in the purification of domestic water. Science World Journal 4: 6-11.

Delelegn A, Sahile S, Husen A (2018) Water purification and antibacterial efficacy of Moringa oleifera Lam Agriculture \& Food Security 7: 1-10. DOI: 10.1186/s40066-018-0177-1.

Feria JJ, Bermudez S, Estrada AM (2014) Eficiencia de la semilla Moringa oleifera como coagulante natural para la remoción de la turbidez del río Sinú. Producción + Limpia 9: 9-22. 
Ferreira VV, Silva IF, Lopes AM, Sant'Anna DO, Lopes A, Mambeli R (2020) Clarification of high-turbidity waters: a comparison of Moringa oleifera and virgin and recovered aluminum sulfate-based coagulants. Environment, Development and Sustainability 22: 4551-4562.

Mani A, Meikandaan TP, Gowrishankar PG, Kanchanabhan TE (2019) A study on treatment of industrial effluent (dyeing) using Moringa oleifera, Tamarina indica as coagulants. International Journal of Civil Engineering and Technology 10: 550-565.

Mendoza I, Fernández N, Ettiene GDA (2000) Uso de la Moringa oleifera como coagulante en la potabilización de las aguas. Ciencia 8: 235-242.

Mera CF, Gutiérrez ML, Montes C, Paz JP (2016) Efecto de la Moringa oleifera en el tratamiento de aguas residuales en El Cauca, Colombia. Biotecnología en el Sector Agropecuario y Agroindustrial 14: 100-109.

Ndabigengesere AS, Narasiah KS (1996) Influence of operating parameters on turbidity removal by coagulation with Moringa oleifera seed. Environmental Technology 17: 1103-1112.

Olson ME, Fahey JW (2011) Moringa oleifera: un árbol multiusos para las zonas tropicales secas. Revista Mexicana de Biodiversidad 82: 1071-1082.

OMS (2017) Progresos en materia de agua potable, saneamiento e higiene: informe de actualización de 2017 y línea de base de los ODS Organización Mundial de la Salud - UNICEF https:/www.who.int/water_sanitation_ health/publications/jmp-2017/es/. Fecha de consulta: 5 de septiembre de 2020.

Rondeau V, Commenges D, Jacqmin-Gadda H, Dartigues JP (2000) Relation between aluminum concentrations in drinking water and Alzheimer's disease: an 8-year follow-up study. American Journal of Epidemiology 152: 59-66.

Rondón M, Díaz Y, Rodríguez S, Guerra B, Fernández E, Tabio D (2017) Empleo de semillas de Moringa oleifera en el tratamiento de residuales liquidos. Ingenieria Hidraulica y Ambiental 38: 87-101.

Sandoval MM, Laines JR (2013) Moringa oleifera una alternativa para sustituir coagulantes metálicos en el tratamiento de aguas superficiales. Ingeniería 17: 93-101.

Santana CR, Pereira DF, Sousa SC, Cavalcanti EB, Silva GF (2010) Evaluation of the process of coagulation/flocculation of produced water using Moringa oleífera Lam. as natural coagulant. Brazilian Journal of Petroleum and Gas 4: 111-117.

Saritha V, Srinivas N, Srikanth NV (2017) Analysis and optimization of coagulation and flocculation process. Applied Water Science 7: 451-460.

Shan TC, Matar AM, Makky EA, Ali EN (2017) The use of Moringa oleifera seed as a natural coagulant for wastewater treatment and heavy metals removal. Applied Water Science 7: 1369-1376.

Sotheeswaran S, Nand V, Matakite M, Kanayathu K (2011) Moringa oleifera and other local seed in water purification in developing countries. Research Journal of Chemistry and Environment 15: 135-138.

SSA (2000) Modificación a la Norma Oficial Mexicana NOM-127-SSA1-1994. Salud ambiental, agua para uso y consumo humano, límites permisibles de calidad y tratamientos a que debe someterse el agua para su potabilización. http://www.salud.gob.mx/unidades/cdi/nom/127ssa14.html. Fecha de consulta: 5 de septiembre de 2020.

Wang Z, Wei X, Yang J, Suo J, Chen J, Liu X, Zhao X (2016) Chronic exposure to aluminium and risk of Alzheimer's disease: a meta-analysis. Neuroscience Letters 610: 200-206. 
Villaseñor D, Astudillo PD, del Real J, Bandala ER (2018) Wastewater treatment using Moringa oleifera Lam seeds: a review. Journal of Water Process Engineering 29: 151-164.

Yin C (2010) Emerging usage of plant-based coagulants for water and wastewater treatment. Process Biochemistry 45: 1437-1444. 